Supporting Information for:

\title{
Automated PET-RAFT Polymerization Towards Pharmaceutical Amorphous Solid Dispersion Development
}

\author{
Rahul Upadhya ${ }^{1}$, Ashish Punia*2, Mythili J. Kanagala ${ }^{1}$, Lina Liu ${ }^{2}$, Matthew Lamm², \\ Timothy A. Rhodes ${ }^{2}$, Adam J. Gormley ${ }^{* 1}$
}

${ }^{1}$ Department of Biomedical Engineering, Rutgers, The State University of New Jersey, Piscataway, NJ 08854, USA

${ }^{2}$ Preformulation Sciences, MRL, Merck \& Co., Inc., Rahway, NJ 07065, USA

Corresponding Authors E-mails: Adam.Gormley@rutgers.edu (Adam J. Gormley)

Ashish.Punia@merck.com (Ashish Punia)

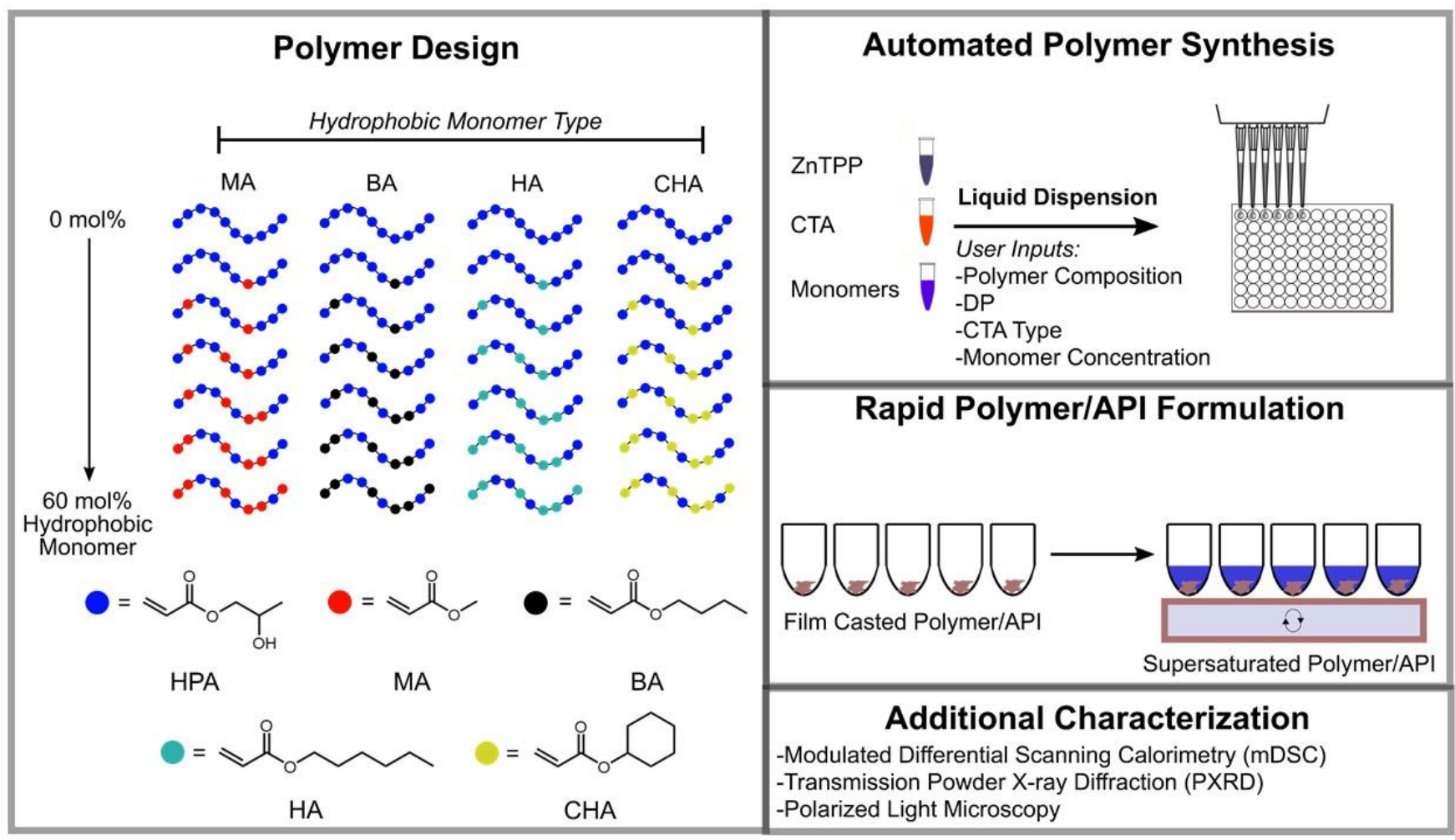




\section{S1. Polymer Structure}

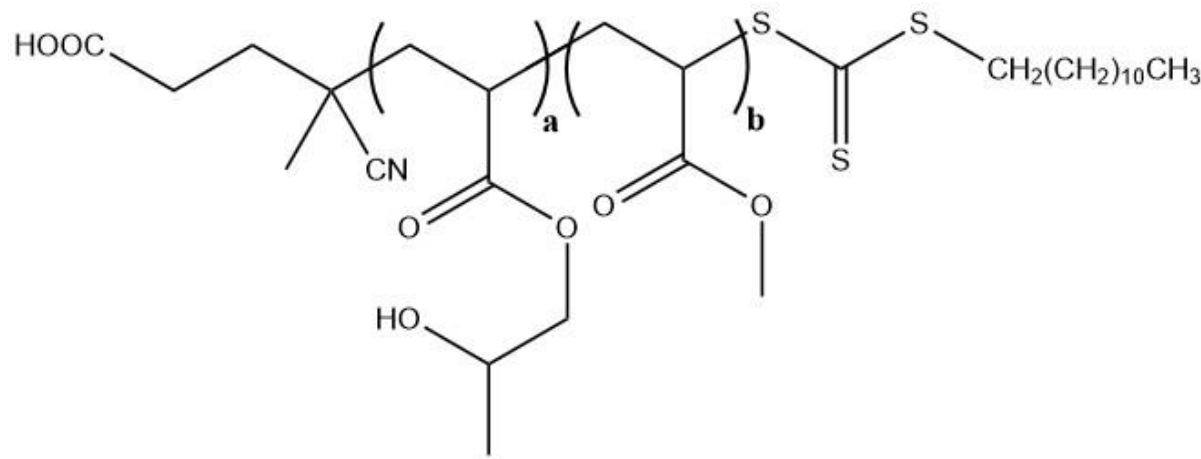

Figure S1. Representative structure of polymer containing chain transfer agent (CTA). Random heteropolymer HPA-MA is displayed, accounting for the presence of CTA involved in PET-RAFT. The CTA used in these experiments was 4-cyano-4-[(dodecylsulfanylthiocarbonyl)sulfanyl] pentanoic acid.

\section{S2. Gel Permeation Chromatography (GPC) Data}

A

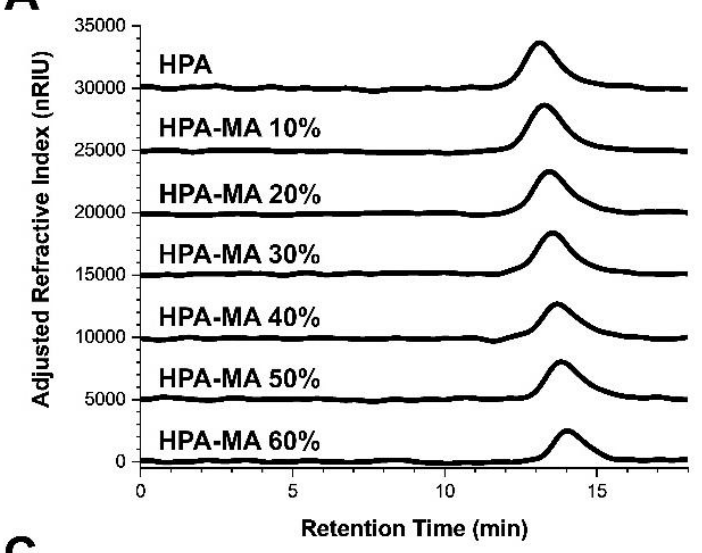

C

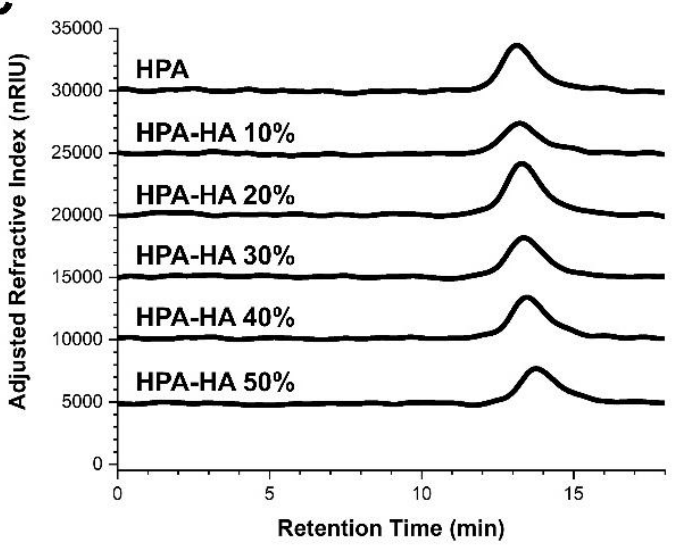

B

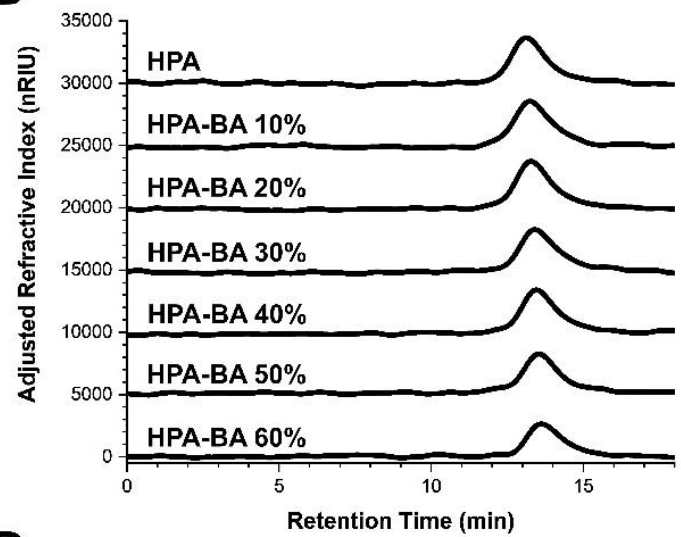

D

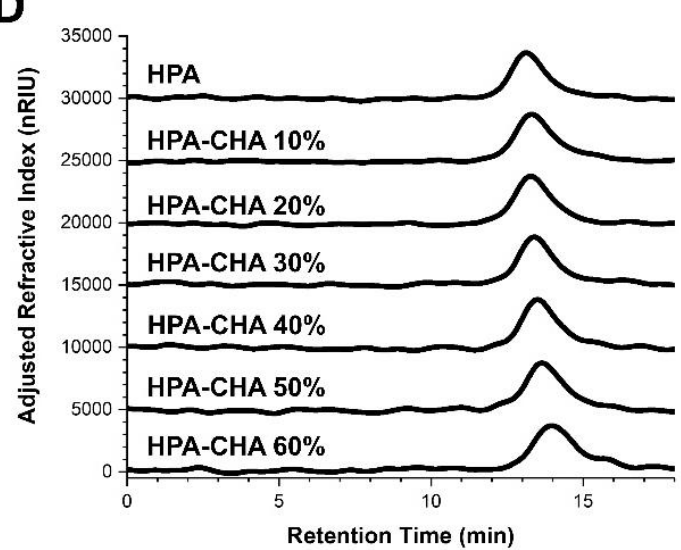

Figure S2. GPC traces of all polymers grouped heteropolymers (A) HPA-MA, (B) HPA-BA, (C) HPAHA, and (D) HPA-CHA containing 10-60 mol\% of MA, BA, HA, and CHA. The GPC trace of HPA homopolymer is displayed alongside all random heteropolymers. Note that the traces were adjusted so they can be stacked. 
Table S1. Signal-to-noise ratio (SNR) corresponding to GPC chromatograms of all polymers.

\begin{tabular}{|c|c|}
\hline Polymer & SNR \\
\hline HPA & 9.12 \\
\hline HPA-MA 10\% & 14.51 \\
\hline HPA-MA 20\% & 15.94 \\
\hline HPA-MA 30\% & 14.51 \\
\hline HPA-MA 40\% & 13.23 \\
\hline HPA-MA 50\% & 9.35 \\
\hline HPA-MA 60\% & 9.56 \\
\hline HPA-BA $10 \%$ & 12.18 \\
\hline HPA-BA $20 \%$ & 16.52 \\
\hline HPA-BA $30 \%$ & 12.63 \\
\hline HPA-BA $40 \%$ & 15.20 \\
\hline HPA-BA $50 \%$ & 14.57 \\
\hline HPA-BA $60 \%$ & 11.44 \\
\hline HPA-HA 10\% & 7.12 \\
\hline HPA-HA 20\% & 15.34 \\
\hline HPA-HA 30\% & 14.81 \\
\hline HPA-HA 40\% & 15.85 \\
\hline HPA-HA 50\% & 11.28 \\
\hline HPA-CHA 10\% & 16.93 \\
\hline HPA-CHA 20\% & 13.39 \\
\hline HPA-CHA 30\% & 9.55 \\
\hline HPA-CHA 40\% & 8.59 \\
\hline HPA-CHA 50\% & 9.85 \\
\hline HPA-CHA $60 \%$ & 7.96 \\
\hline
\end{tabular}

SNR was determined by comparison of the maximal peak signal intensity (10-17 min) with the greatest fluctuation in the baseline region (0-10 $\mathrm{min})$. 


\section{S3. Ultra-Performance Liquid Chromatography (UPLC) Data}

A

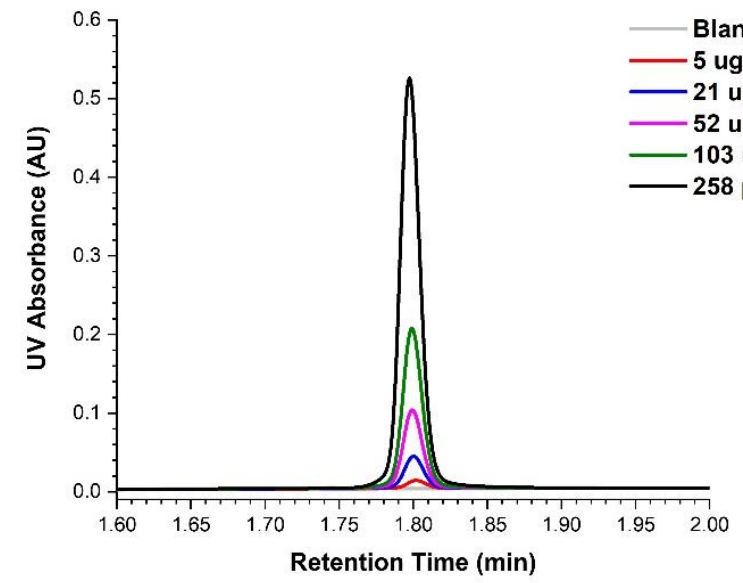

B

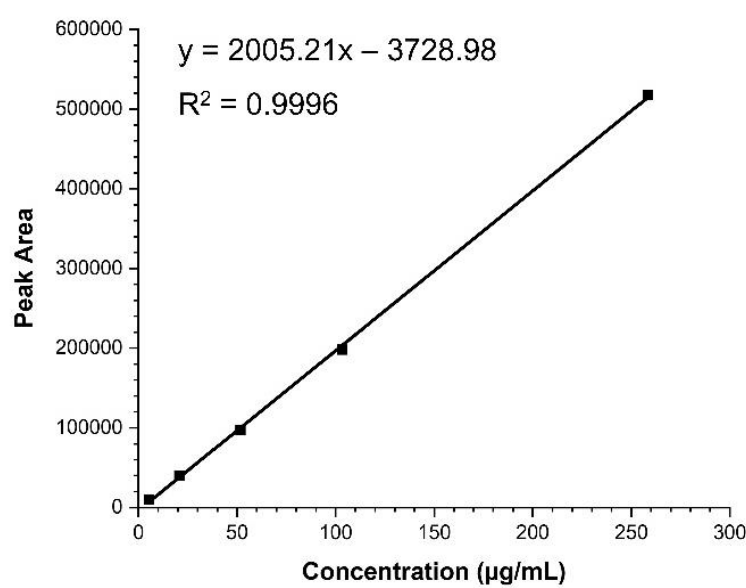

Figure S3. UPLC standard injections of probucol. (A) Various concentrations of probucol (0, 5.2, 20.7, 51.7, 103.4, and 258.6 $\mu \mathrm{g} / \mathrm{mL}$ ) were prepared directly in 40:40:20 MeOH:MeCN: $\mathrm{H}_{2} \mathrm{O}$ and injected as concentration standards for UPLC analysis. The method ran for a total of $2.50 \mathrm{~min}$ and all peaks were integrated using the UV detection wavelength of $254 \mathrm{~nm}$. The peak displayed at about $1.80 \mathrm{~min}$ represents probucol. (B) Linear fit was done of the area under the curve for subsequent concentration determination. This linear fit results in this well-fit line of best fit $\left(\mathrm{R}^{2}=0.9996\right)$. 
A

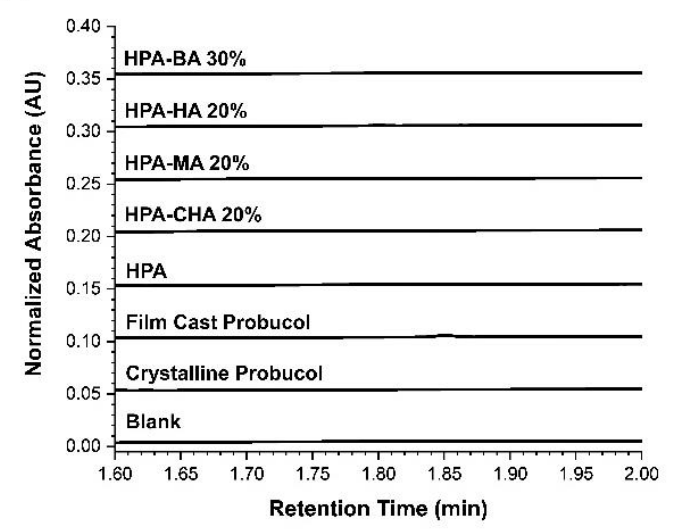

B

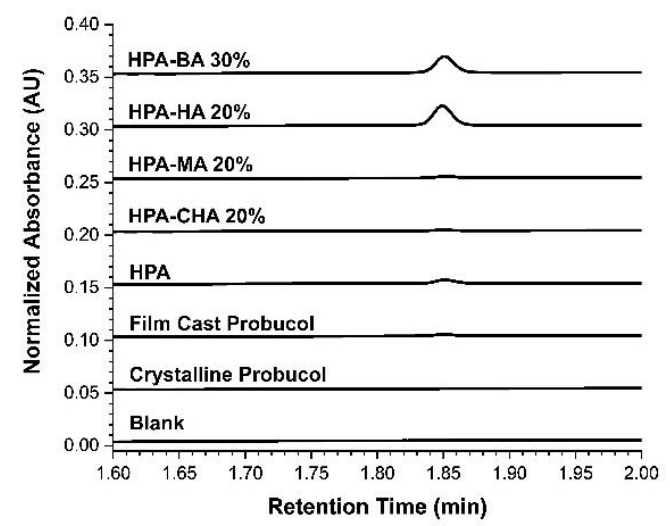

Figure S4. Dissolution effect of synthesized polymers evaluated by the film casting screen and UPLC. (A) Normalized UV absorbance of synthesized polymers (HPA, HPA-CHA 20\%, HPA-MA 20\%, HPAHA 20\%, and HPA-BA 30\%), probucol, and blank. Probucol was prepared by directly dissolving into biorelevant media (crystalline probucol) or by film casting in acetone before dissolving into biorelevant media (film cast probucol). These plots were taken at the earliest time point into the film casting assay (15 min) using a UV detection wavelength of $254 \mathrm{~nm}$. There are typically no important peaks visualized at this early time point. (B) Normalized UV absorbance taken at the last time point (330 min) of the film casting assay at $254 \mathrm{~nm}$. The peak corresponding to the probucol peak (1.85 min) can be visualized for some samples better than others. For display purposes, normalization was done to fit chromatograms on the same set of axes by vertically translating chromatograms.

A

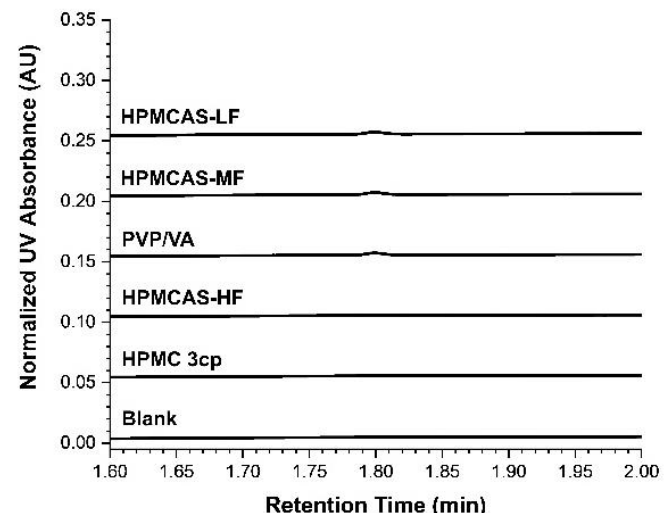

B

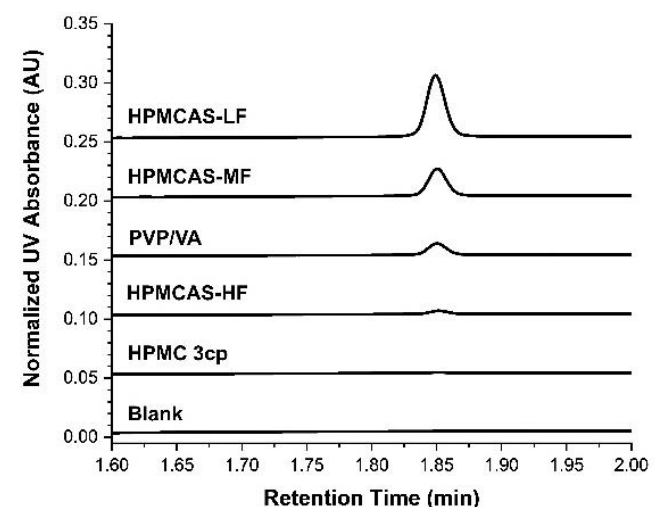

Figure S5. Dissolution effect of standard polymer excipients evaluated by the film casting screen and UPLC. (A) Normalized UV absorbance of standard polymer excipients and blank. The standard polymer excipients are vinyl pyrrolidone-vinyl acetate copolymer (PVP/VA), hydroxypropyl methylcellulose acetate succinate (HPMCAS), and hydroxypropyl methylcellulose (HPMC). These plots were taken at the earliest time point into the film casting assay $(15 \mathrm{~min})$ using a UV detection wavelength of $254 \mathrm{~nm}$. (B) Normalized UV absorbance taken at the last time point (330 min) of the film casting assay at 254 $\mathrm{nm}$. The peak corresponding to the probucol peak $(1.85 \mathrm{~min})$ can be visualized for some samples better than others. For display purposes, normalization was done to fit chromatograms on the same set of axes by vertically translating chromatograms. 


\section{S4. Modulated Differential Scanning Calorimetry (mDSC) Data}

A

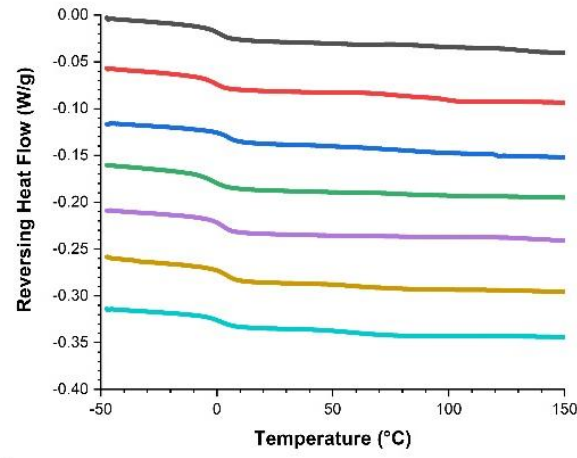

C

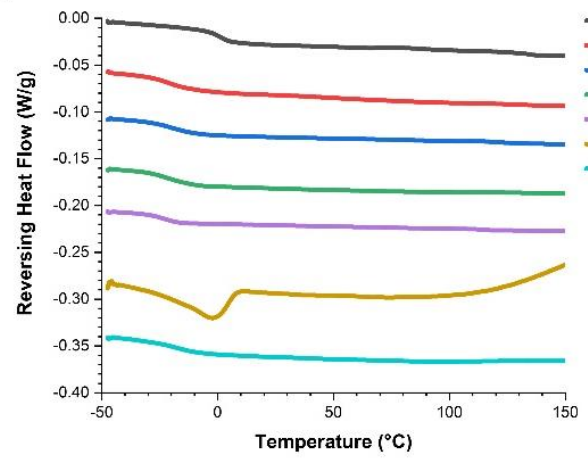

B
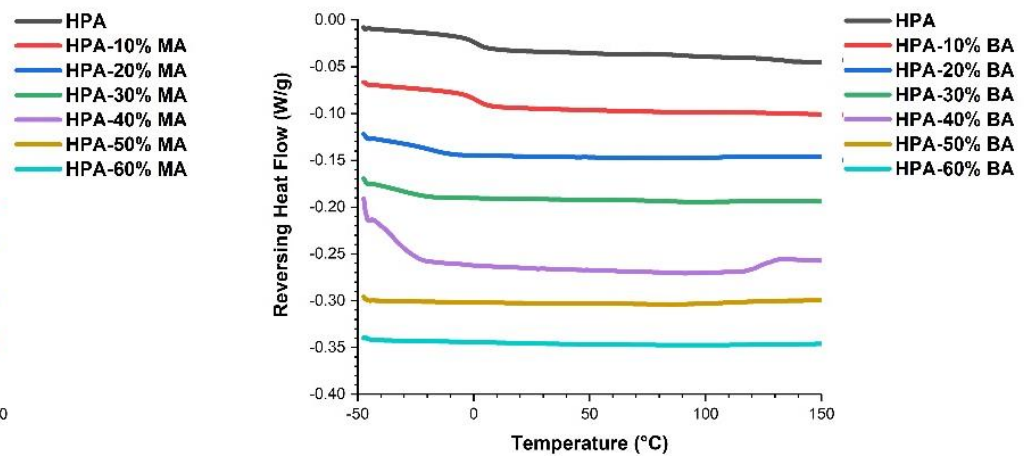

D

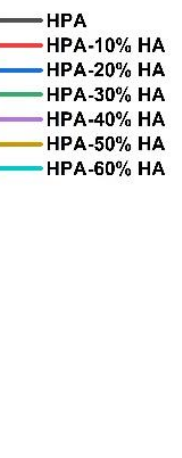

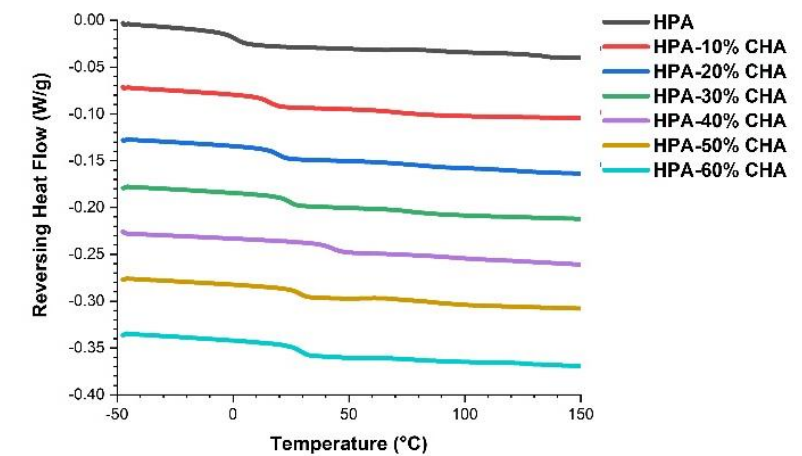

Figure S6. Reversing heat flow mDSC of synthesized polymers (first heat cycle). Synthesized polymers grouped by hydrophobic comonomer (MA, BA, HA, and CHA) were weighed out to at least $5 \mathrm{mg}$ and analyzed by mDSC. Reversing heat flow plots are for (A) MA, (B) BA, (C) HA, and (D) CHA comonomers. For display purposes, normalization was completed to translate reversing heat flow plots vertically and stack. 
A

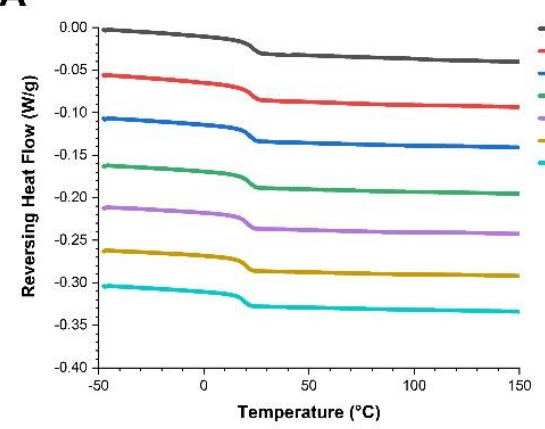

\section{C}

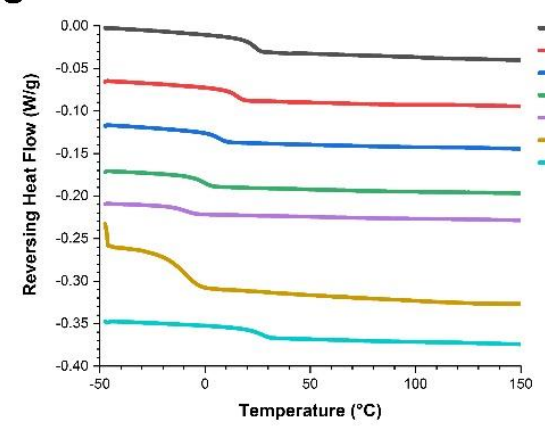

B

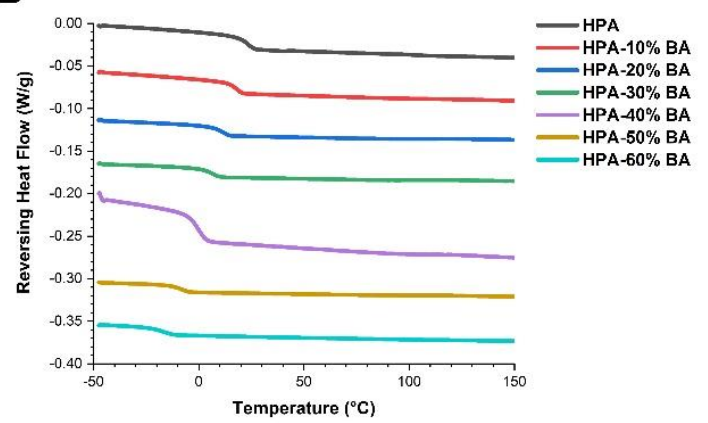

D

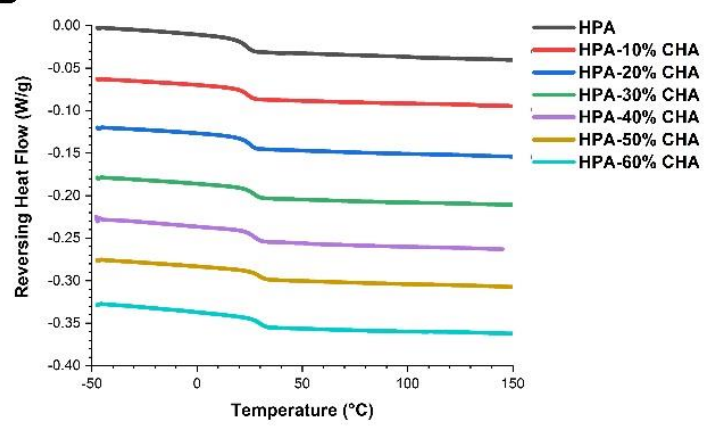

Figure S7. Reversing heat flow mDSC of synthesized polymers (second heat cycle). Synthesized polymers grouped by hydrophobic comonomer (MA, BA, HA, and CHA) were weighed out to at least $5 \mathrm{mg}$ and analyzed by mDSC. Reversing heat flow plots are for (A) MA, (B) BA, (C) HA, and (D) CHA comonomers. For display purposes, normalization was completed to translate reversing heat flow plots vertically and stack. 
A

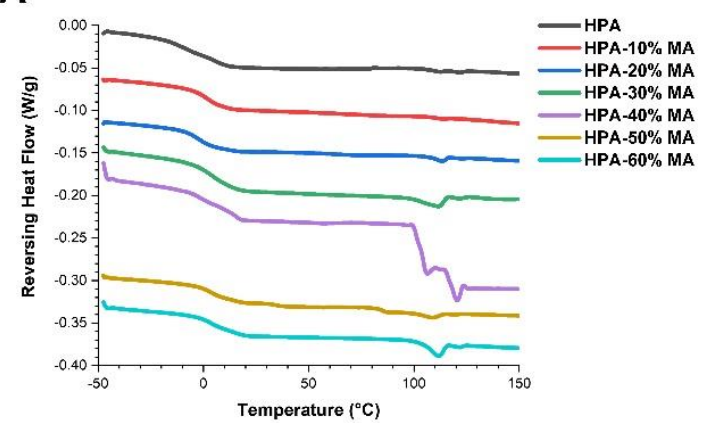

C

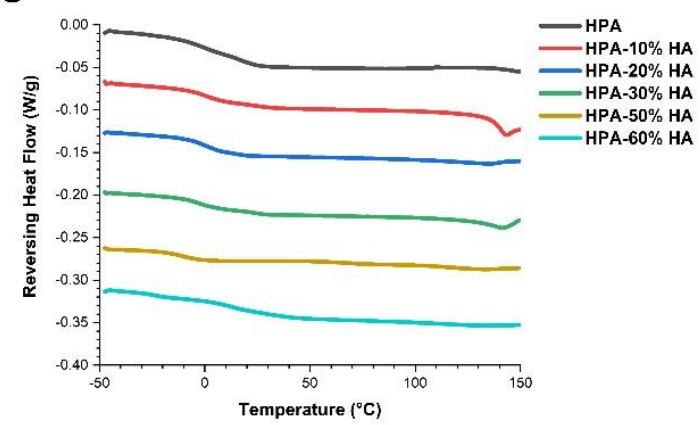

B

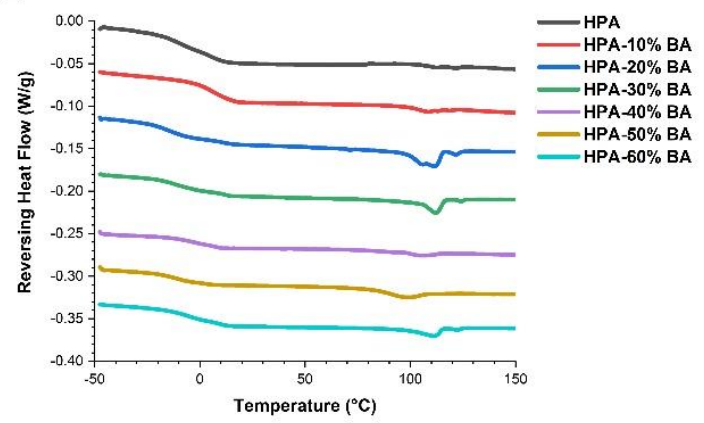

D

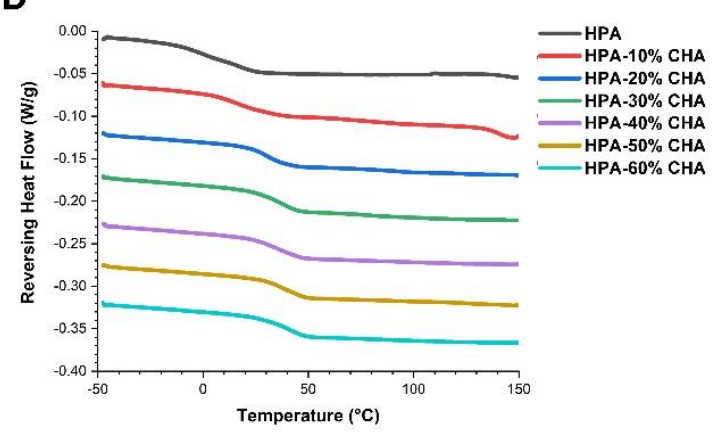

Figure S8. Reversing heat flow mDSC of synthesized polymer/probucol casted films (first heat cycle). Approximately $2 \mathrm{mg}$ of material (in acetone) was dropped in corresponding pans and left to dry overnight. Reversing heat flow plots are for (A) MA, (B) BA, (C) HA, and (D) CHA comonomer/API films. Each sample contains $20 \mathrm{wt} \%$ probucol. For display purposes, normalization was completed to translate reversing heat flow plots vertically and stack. Note that HPA-40\% HA could not be analyzed due to not containing a distinct $\mathrm{T}_{\mathrm{g}}$ region. This could be a result of miscibility issues. 
A

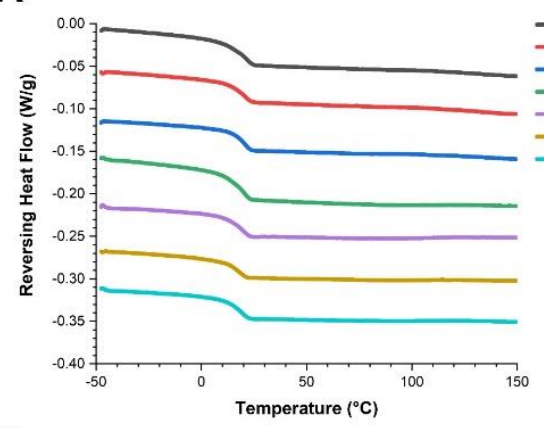

C

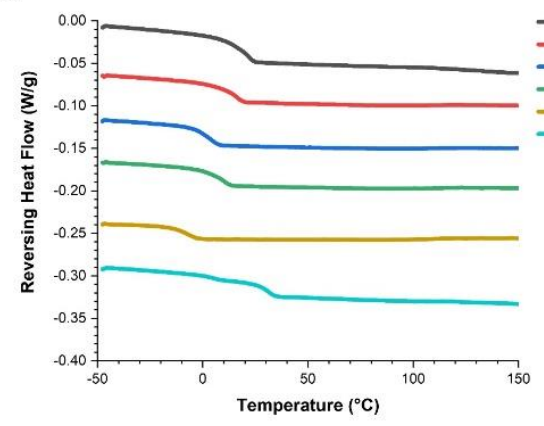

B

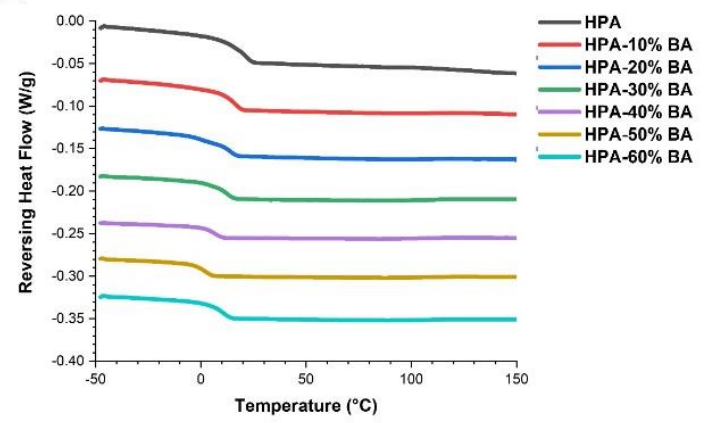

D

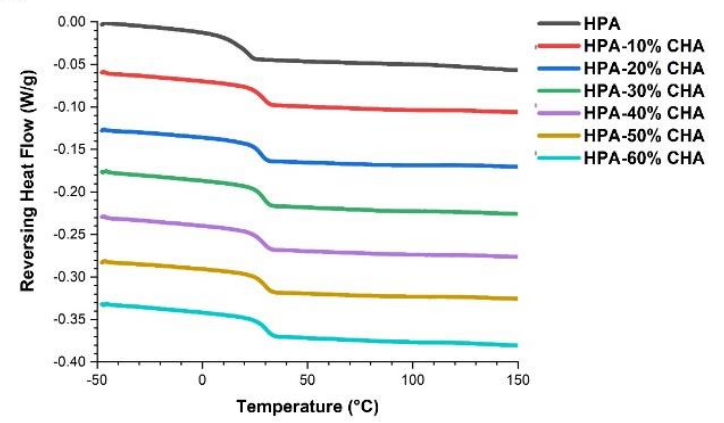

Figure S9. Reversing heat flow mDSC of synthesized polymer/probucol casted films (second heat cycle). Approximately $2 \mathrm{mg}$ of material (in acetone) was dropped in corresponding pans and left to dry overnight. Reversing heat flow plots are for (A) MA, (B) BA, (C) HA, and (D) CHA comonomer/API films. Each sample contains $20 \mathrm{wt} \%$ probucol. For display purposes, normalization was completed to translate reversing heat flow plots vertically and stack. Note that HPA-40\% HA could not be analyzed due to not containing a distinct $\mathrm{T}_{\mathrm{g}}$ region. This could be a result of miscibility issues.

A

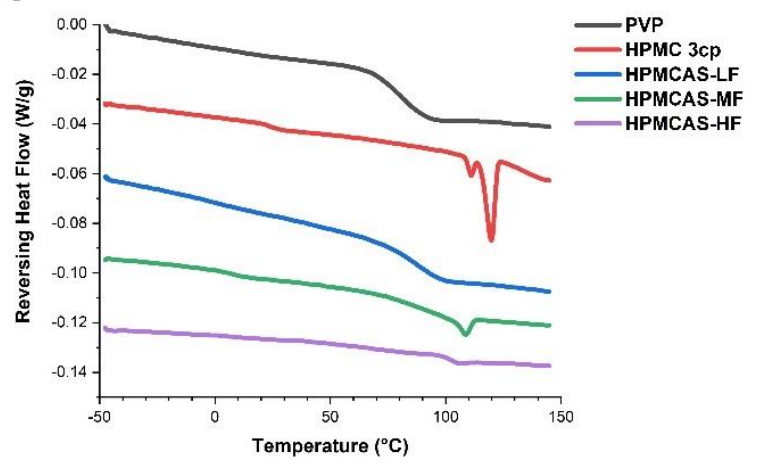

B

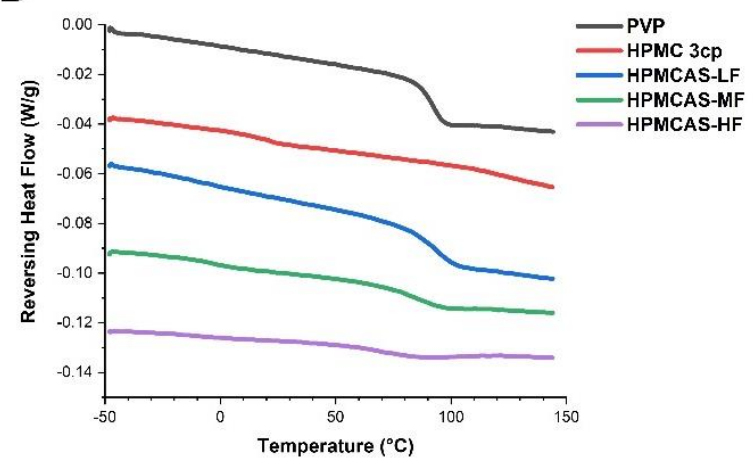

Figure S10. Reversing heat flow mDSC of standard polymer/probucol casted films. These standard polymers include PVP/VA, HPMC 3cp, HPMCAS-LF, HPMCAS-MF, and HPMCAS-HF. Approximately $2 \mathrm{mg}$ of material was dropped in corresponding pans and left to dry overnight. Reversing heat flow plots are for the (A) first heat cycle and (B) second heat cycle. Each sample contains $20 \mathrm{wt} \%$ probucol. For display purposes, normalization was completed to translate reversing heat flow plots vertically and stack. 
Table S2. Analyzed $\mathrm{T}_{\mathrm{g}}$ values of polymer/probucol casted films, as determined by mDSC half height.

\begin{tabular}{|c|c|}
\hline Polymer & $\mathrm{T}_{\mathrm{g}}\left({ }^{\circ} \mathrm{C}\right)$ \\
\hline HPA & 18.9 \\
\hline HPA-10\% MA & 19.4 \\
\hline HPA-20\% MA & 19.4 \\
\hline HPA-30\% MA & 18.1 \\
\hline HPA- $40 \%$ MA & 17.5 \\
\hline HPA-50\% MA & 17.3 \\
\hline HPA-60\% MA & 17.6 \\
\hline HPA-10\% BA & 15.3 \\
\hline HPA-20\% BA & 11.1 \\
\hline HPA-30\% BA & 10.3 \\
\hline HPA-40\% BA & 6.3 \\
\hline HPA-50\% BA & 1.2 \\
\hline HPA-60\% BA & 8.3 \\
\hline HPA-10\% HA & 13.8 \\
\hline HPA-20\% HA & 2.0 \\
\hline HPA-30\% HA & 8.0 \\
\hline HPA-40\% HA & $N / A$ \\
\hline HPA-50\% HA & 8.5 \\
\hline HPA-60\% HA & $\mathrm{N} / \mathrm{A}$ \\
\hline HPA-10\% CHA & 21.9 \\
\hline $\mathrm{HPA}-20 \% \mathrm{CHA}$ & 26.9 \\
\hline $\mathrm{HPA}-30 \% \mathrm{CHA}$ & 28.8 \\
\hline $\mathrm{HPA}-40 \% \mathrm{CHA}$ & 28.1 \\
\hline HPA-50\% CHA & 29.0 \\
\hline HPA-60\% CHA & 29.8 \\
\hline PVP & 96.0 \\
\hline HPMC $3 c p$ & $N / A$ \\
\hline HPMCAS-LF & 98.3 \\
\hline HPMCAS-MF & N/A \\
\hline HPMCAS-HF & $N / A$ \\
\hline
\end{tabular}

*N/A: $\mathrm{T}_{\mathrm{g}}$ region was too broad or indistinguishable, likely due to varying degrees of immiscibility. 


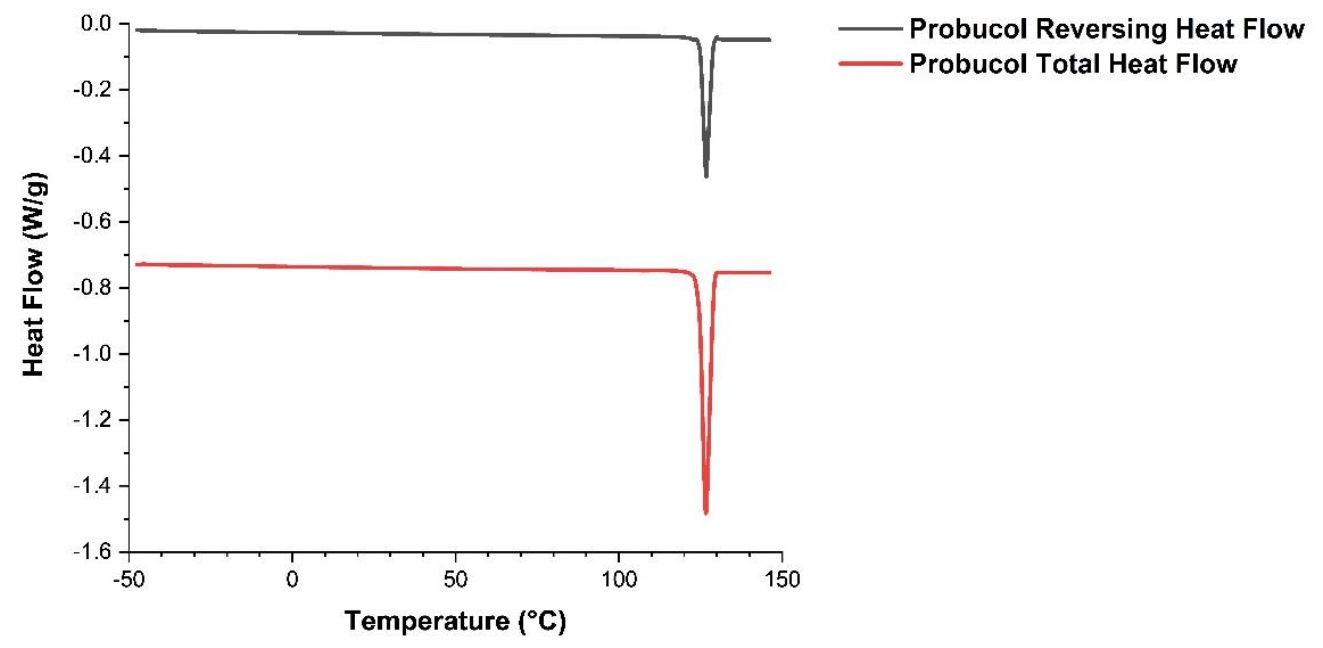

Figure S11. Reversing heat flow and total heat flow determined by mDSC of probucol. Approximately $5 \mathrm{mg}$ of probucol was weighed out directly into the DSC pan. The measured melting temperature $\left(\mathrm{T}_{\mathrm{m}}\right)$ was about $126^{\circ} \mathrm{C}$. For display purposes, normalization was completed to translate reversing heat flow plots vertically and stack.

\section{S5. NMR}

All synthesized polymers (random heteropolymers containing HPA and hydrophobic monomers MA, BA, HA, and CHA) were further characterized by ${ }^{1} \mathrm{H}$ NMR spectroscopy. All experiments were conducted on a Bruker Avance Neo $500 \mathrm{MHz}$ liquid state NMR instrument at a temperature of $25^{\circ} \mathrm{C}$. This instrument is equipped with $2 \mathrm{RF}$ channels, an RT-DR-BF/1H-5mmZ-SP iProbe, and an autosampler. ${ }^{1} \mathrm{H}$ NMR was utilized to elucidate the polymer conversion and experimental monomer incorporation by end-group analysis. End-group analysis enables researchers to determine if actual monomer incorporation varies from the feed ratio.

To determine conversion and the number average molecular weight $\left(\mathrm{M}_{\mathrm{n}}\right)$, end-group analysis was completed. As described previously and shown in Figure S4, the CTA used for polymer synthesis was 4-cyano-4-[(dodecylsulfanylthiocarbonyl)sulfanyl]pentanoic acid. The proton integration corresponding to the CTA end-group at $0.84-0.87 \mathrm{ppm}$ for $\underline{\mathrm{CH}_{3}}-\left(\mathrm{CH}_{2}\right)_{11}-\mathrm{S}(3 \mathrm{H})$ was assessed alongside characteristic proton integrations for HPA at 4.76ppm for $\mathrm{O}-\mathrm{CH}_{2}-\mathrm{CH}_{-}-\mathrm{CH}_{3}$ $\mathrm{OH}(2 \mathrm{H}), \mathrm{MA}$ at $3.56 \mathrm{ppm}$ for $\mathrm{O}-\underline{\mathrm{CH}_{3}}(3 \mathrm{H}), \mathrm{BA}$ at $1.31 \mathrm{ppm}$ for $\mathrm{O}-\mathrm{CH}_{2}-\left(\mathrm{CH}_{2}\right)_{2}-\mathrm{CH}_{3}(4 \mathrm{H}), \mathrm{HA}$ at 1.27ppm for $\mathrm{O}-\mathrm{CH}_{2}-\left(\mathrm{CH}_{2}\right)_{4}-\mathrm{CH}_{3}(8 \mathrm{H})$, and $\mathrm{CHA}$ at 4.64ppm for $\mathrm{O}-\mathrm{CH}(1 \mathrm{H})$. A mesitylene internal standard was included at 6.77ppm. Representative spectra are included in Figures S15S18. 

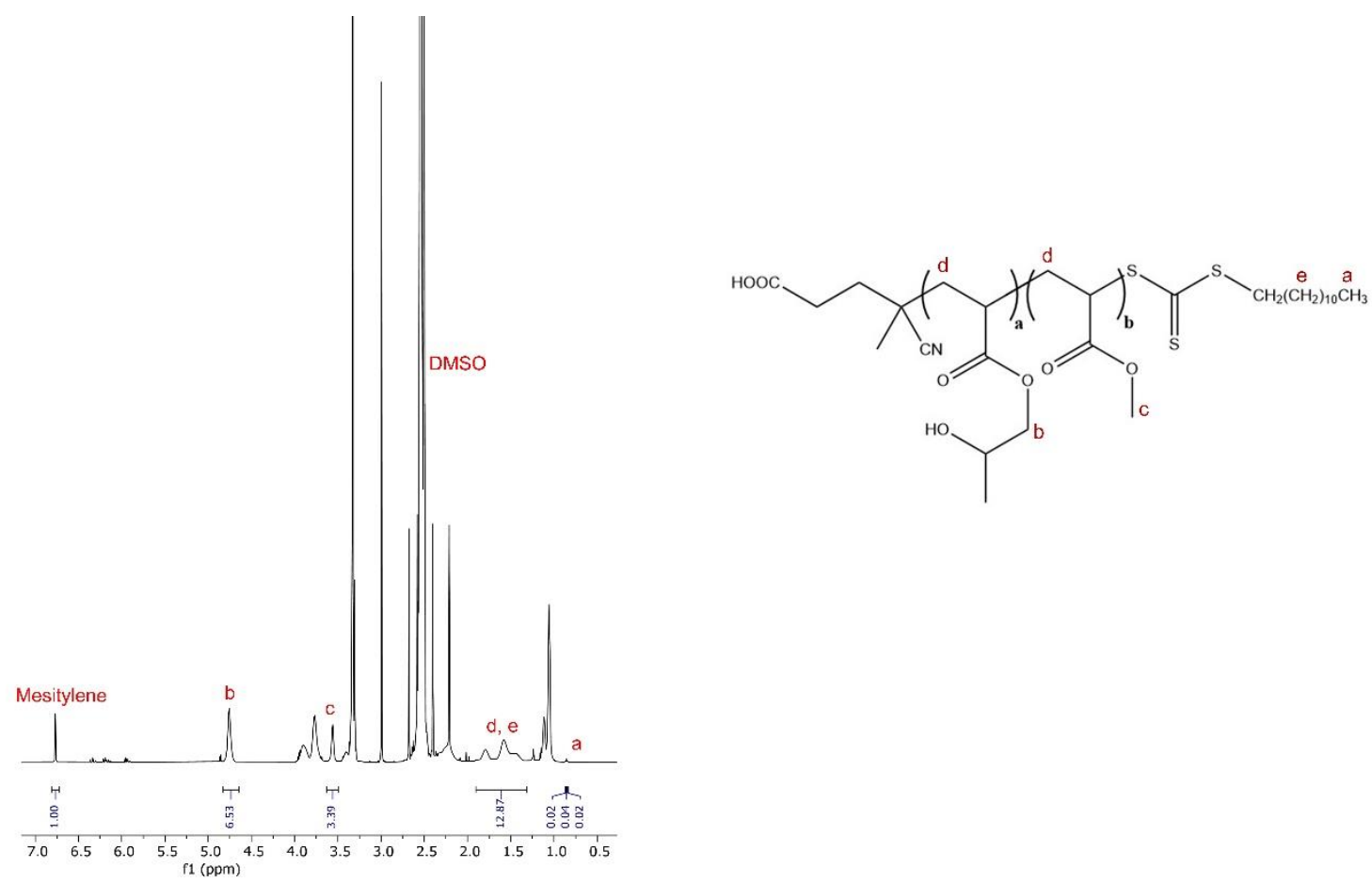

Figure S12. ${ }^{1} \mathrm{H}$ NMR of random heteropolymer HPA-MA 20\%. The polymer structure is also displayed.

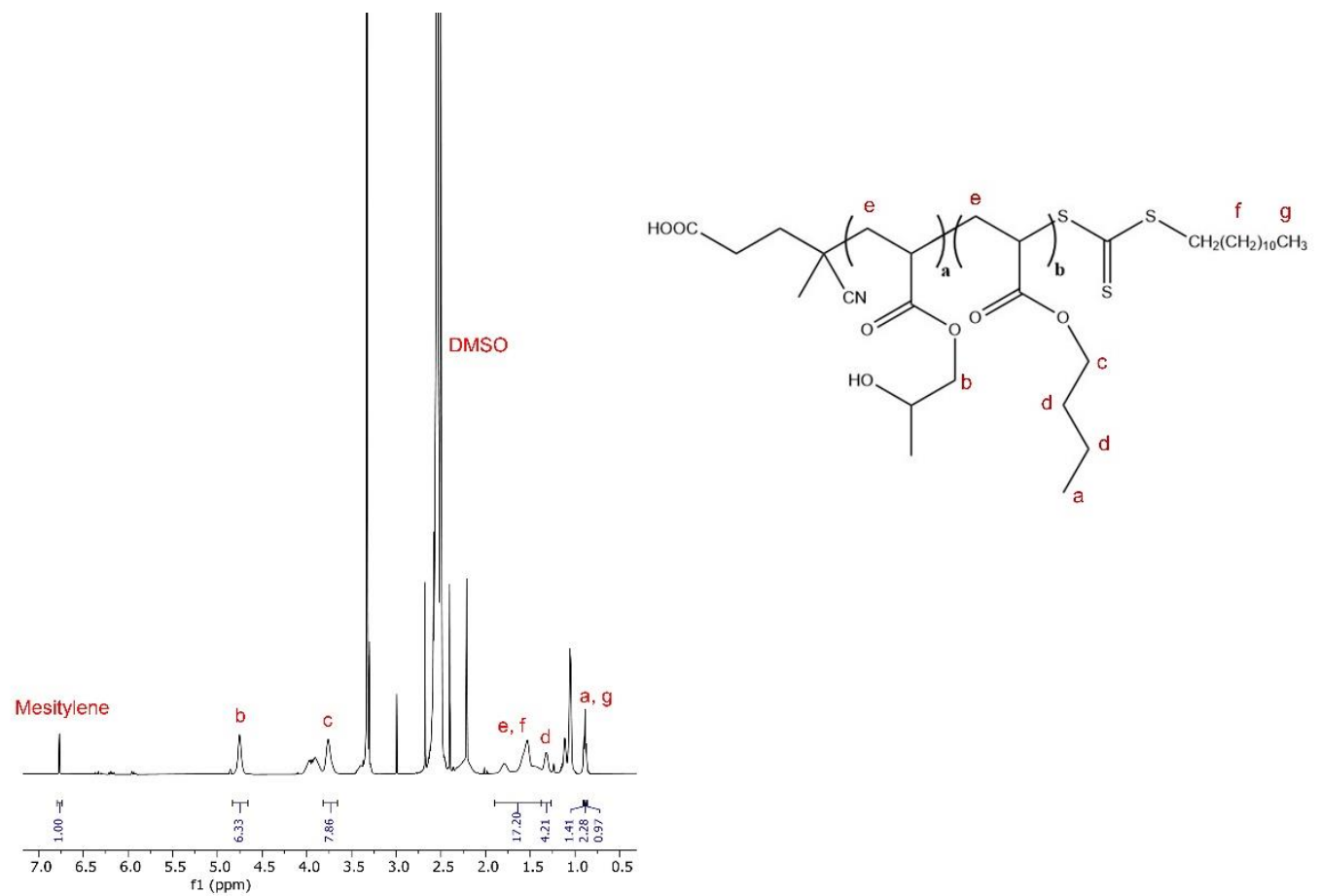

Figure S13. ${ }^{1} \mathrm{H}$ NMR of random heteropolymer HPA-BA $20 \%$. The polymer structure is also displayed. 

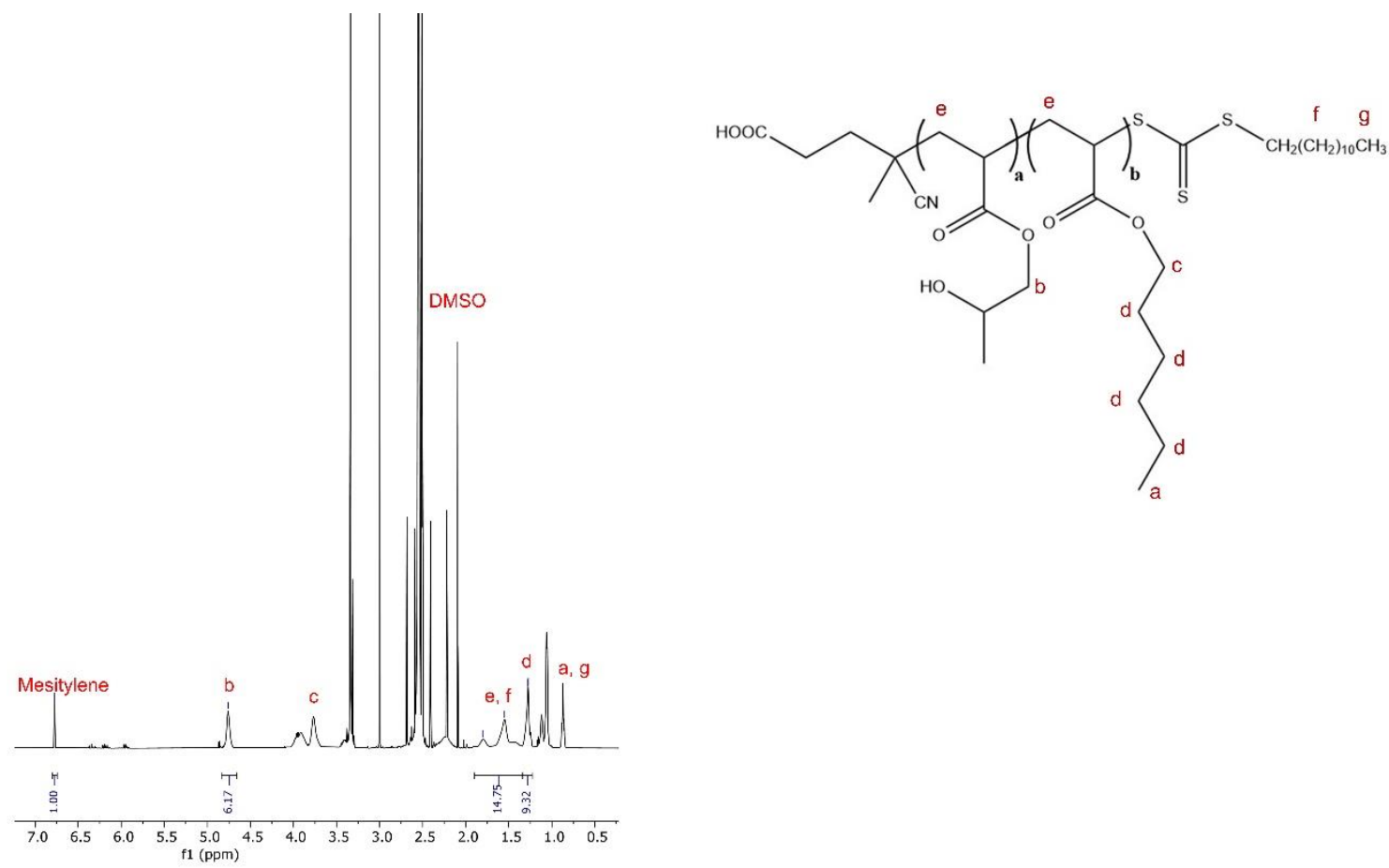

Figure S14. ${ }^{1} \mathrm{H}$ NMR of random heteropolymer HPA-HA 20\%. The polymer structure is also displayed.

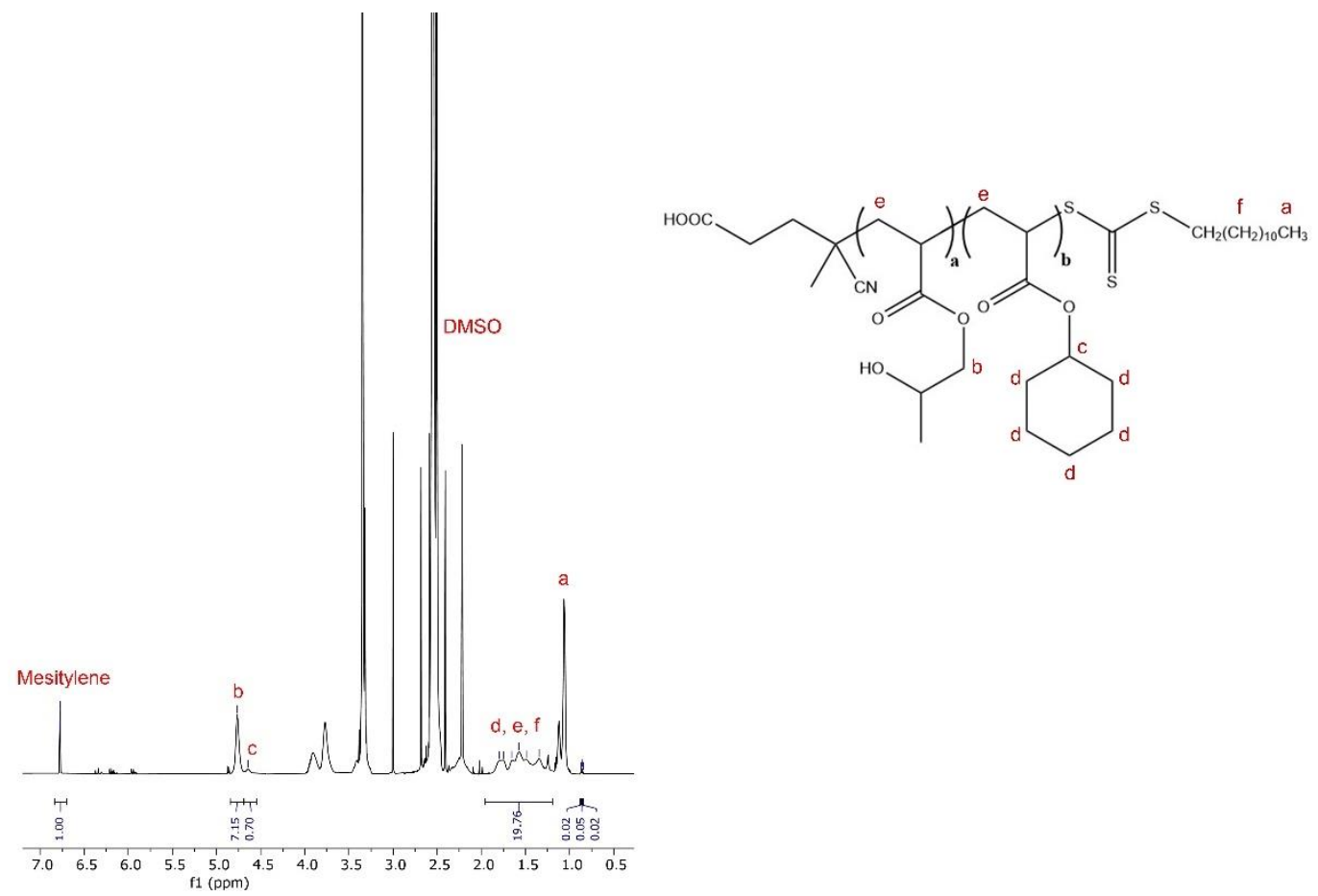

Figure S15. ${ }^{1} \mathrm{H}$ NMR of random heteropolymer HPA-CHA $20 \%$. The polymer structure is also displayed. 